\title{
Online Neural Architecture Search (ONAS): Adapting neural network architecture search in a continuously evolving domain. [Proposal]
}

\author{
Nathan Buskulic \\ nathan.buskulic@uni-siegen.de \\ University of Siegen, Dept. of \\ Computer Science \\ Siegen, Germany
}

\author{
Edward Bergman \\ Edward.Bergman@uni-siegen.de \\ University of Siegen, Dept. of \\ Computer Science \\ Siegen, Germany
}

\author{
Joeran Beel \\ joeran.beel@uni-siegen.de \\ University of Siegen, Dept. of \\ Computer Science \\ Siegen, Germany
}

\begin{abstract}
Neural Architecture Search research has been limited to fixed datasets and as such does not provide the flexibility needed to deal with real-world, constantly evolving data. This is why we propose the basis of Online Neural Architecture Search (ONAS) to deal with complex, evolving, data distributions. We formalise ONAS as a minimisation problem upon which both the weights and the architecture of the neural network needs to be optimised for the data up until a time $t_{i}$. To solve this problem, we adapt a DARTS optimisation process, associated with an early stopping scheme, by using the supernet optimised on previous data as a warm-up initial state. This allows the architecture of the neural network to evolve as the data distribution evolves while limiting the computational burden. This work aims at building the initial mathematical formalism of the problem as well as the development of a framework where NAS methods could be used to solve this problem. Finally, several possible next steps are presented to show the potential of this field of Online Neural Architecture Search.
\end{abstract}

\section{INTRODUCTION}

We have recently seen a renewed interest in the domain of neural architecture search (NAS) $[2-8,12,15,16,18,19,21-23,25-28$, 30-40] after these methods obtained state-of-the-art results on different classical tasks of Deep Learning. The interest in NAS is two-fold. It alleviates the burden of neural network architecture design from experts while also providing mathematically founded ways of designing neural network architectures adapted to specific tasks and datasets.

However, NAS research has been focused on optimising the architecture of neural networks for datasets with static distributions without taking interest in a more real-world like, online approach. That is, a context in which data comes sequentially through time with a possible evolution of the distribution underlying the data. While online data processing is a topic that received a lot of interest $[1,10,14]$, no work has yet being done, to the best of our knowledge, to adapt the architecture of neural networks in an online setting. This context of data streams is what most in-production machine learning systems have to deal with and having NAS methods adapted to this context is therefore of major importance since redoing NAS methods from scratch is often practically impossible due to computational constraints.

\section{RESEARCH PROBLEM}

Real-world data is changing constantly and many algorithms are not built to deal with this problem. Most algorithms only train on a set of data taken up to an instant $t_{0}$ and are then applied to new data points at instants $t_{1}, \ldots, t_{n}$. This widely used scheme makes the assumption that the underlying distribution of the data will not change significantly between $t_{0}$ and $t_{n}$. While this assumption can hold in a laboratory, for in-production systems such as autonomous cars, this assumption will not hold. For example, the car can be used in one country and travel through different countries where the car models and the road types might be very different.

In the case of Deep Learning, the solution chosen to mitigate this problem is often a re-training of the network with the new data and part of the older data. This can be combined with the use of transfer learning to only fine tune the last layers of a complex network and keep the knowledge it learned from previous data. However, this approach is insufficient when the distribution changes in complexity and the previous architecture has not enough expressiveness to catch the shift in the distribution.

This fact is demonstrated in Figure 1 where a very simple network with one hidden neuron would have a perfect score on the first dataset but it would fail when presented with the second dataset. In this case, a change in the architecture of the network is necessary to make it able to catch the distribution of the new data. While this example is very simple, it is easy to see how it can generalise to more complex cases. NAS is therefore a good solution to this problem but the high incurring cost of these methods makes it impossible to use often, like it is being classically done with re-training. Thus, we argue that developing methods able to deal with evolving data distributions in an online fashion is of major importance for real-world use cases of deep learning algorithms.

\section{RESEARCH GOAL}

Our goal is to create a new strategy to enable NAS to adapt to changing data distributions without the computational burden of doing the whole NAS process again. This can be understood as bringing the benefit of transfer learning to NAS methodology. This online NAS algorithm would lead to a drastic reduction in computation when new data is observed while also producing state of the art performances when compared to redoing the entire NAS process several times. The difference between the two methods should be most visible when the change in the data distribution is significant. 


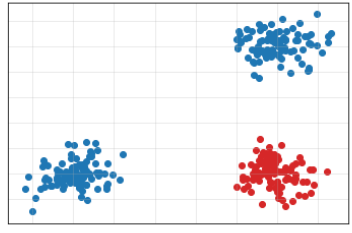

Data distribution at $t_{0}$

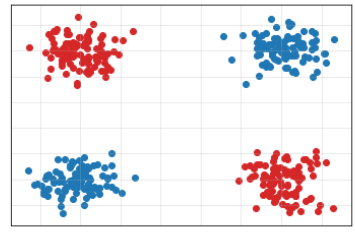

Data distribution at $t_{1}$
Figure 1: A data distribution evolving between time $t_{0}$ and time $t_{1}$. At time $t_{0}$, a neural network with one neuron can separate the two classes while the distribution at $t_{1}$ needs a more expressive network to correctly separate the classes.

Indeed, while ONAS would adapt to the changing distribution, traditional NAS methods would not catch that evolution and therefore provide less meaningful results for distributions changing quickly.

\section{RELATED WORK}

Traditional Neural Architecture Search methods uses either reinforcement learning with meta controllers [2, 11, 13, 25], evolutionary algorithms $[8,9,21,24,29,36]$, or differentiable frameworks $[3,5,12,15,16,18,32-35]$. While the first NAS methods required an excessive amount of computing power (3000 GPU days) [2, 26], newer methods reduced this burden by 3 orders of magnitudes and allowed the development of NAS for a wide range of tasks $[3,11,18]$. Even if a lot of work has been put into reducing the calculation and memory burden of these methods, they are still expensive to compute and often use a search space already good for a specific task. Differentiable methods are the quickest to compute which makes them more interesting to use in a context where we need to do this search often, due to the online nature of the problem. As of now, however, NAS research has not been interested in the idea of online data streams and has focused solely on the context of optimising for a single, static, dataset.

On the other hand, research about online data streams has been flourishing but only a few works are interested in the evolution of neural networks properties other than network weights. In the field of continuous learning, some work uses neural architecture search to make the architecture of the neural network evolve as new classes or new tasks need to be taken into account [11,13]. These methods, however, confines the evolution of a data distribution to the apparition of new classes or new tasks. It does not take into account the evolution of the data from a given class through time.

The work closest to ours is Liu et al [17] which takes interest in how to train a deep learning model to be able to adapt to an evolutionary distribution through the use of a trainable domain adaptor module. While they also focus on the problem of evolving distributions, their work is heavily rooted in the idea of domain adaptation. As such, they do not make the architecture evolve but they train a module to adapt the new data to the existing model. They defined the fundamental formalism of this question but do not answer it through neural architecture changes and prefer a domain transfer point of view. This approach does not fit our problem since they make the assumption that the complexity of the distribution will not change which is not true in our case. This is why we need to be able to adapt the architecture of the neural network and not only the data used in our algorithm.

\section{ONLINE NEURAL ARCHITECTURE SEARCH (ONAS)}

We propose to adapt neural architecture search algorithms to an online data stream context to deal with an evolving distribution of data. To capture the underlying shift in a distribution of data, an adaptive architecture should be used. These adapted architectures also mean an optimisation of the number of weights to optimise and thus, a more efficient training for the provided number of data points.

We express ONAS as a minimisation problem close to the one from Liu et al [17] where we optimise a model $f$ parametrised not only by a vector of weights $\theta$ but also by a vector of architecture parameters $\alpha$ which allows us to frame this as a NAS problem i.e.

$$
\min _{\theta, \alpha} \mathbb{E}_{t \sim U(0,1)} \mathbb{E}_{(x, y) \sim Q_{t}} L\left(f\left(x, \theta_{t}, \alpha_{t}\right), y\right)
$$

The distribution at time $t$ is denoted by $Q_{t}, U$ is the uniform distribution and $L$ is a loss function. The data comes sequentially in unlabelled batches :

$$
T=\left\{\mathbf{X}_{t_{0}}, \mathbf{X}_{t_{1}}, \ldots, \mathbf{X}_{t_{n}}\right\}
$$

For each batch, we consider that a variable amount of data is labelled to be able to adapt the algorithm to the evolving data distribution. Contrary to the work of Liu et al [17] we do not have memory constraints and we can thus use data from previous batches to overcome the problem of catastrophic forgetting and keep the knowledge the network learned previously.

To optimise this problem we will use an adapted NAS algorithm derived from DARTS [18] using partial channel connection [34] and an early stopping scheme [38] for increase stability. We denote the optimisation process to find the best $\theta$ and $\alpha$ vectors as the following function :

$$
\theta, \alpha=\operatorname{DARTS}\left(\mathbf{X}_{t_{0}}, \theta_{t_{0}}, \alpha_{t_{0}}\right)
$$

where $\mathrm{X}$ is the training dataset, $\theta_{t_{0}}$ is the initial value of the $\theta$ vector and $\alpha_{t_{0}}$ is the initial value of the $\alpha$ vector used in the optimisation process. We formalise the supernet found by the DARTS optimisation as $s(\theta, \alpha)$ and the discretisation of the supernet as $f(\theta, \alpha)$.

If we assume that at time $t_{i}$ we have an optimised $s\left(\theta_{i}, \alpha_{i}\right)$ and $f\left(\theta_{i}, \alpha_{i}\right)$, we can calculate the next architecture by applying the DARTS optimisation scheme, using the previously optimised parameters as a warm-up initial state. Formally we have :

$$
\theta_{t_{i+1}}, \alpha_{t_{i+1}}=\operatorname{DARTS}\left(\mathbf{X}_{t_{0}, \ldots, t_{i+1}}, \theta_{t_{i}}, \alpha_{t_{i}}\right)
$$

with $\theta_{t_{i}}$ being the optimised vector of $\theta$ using data up to time $t_{i}$. This process is shown in Figure 2. If we assume that we can have enough data at time $t_{0}$ to find a good initial architecture, then by applying the optimisation process iteratively with new data, we should be able to obtain an optimised neural network architecture able to 
evolve through time while limiting the computational burden once the first iteration has been calculated.

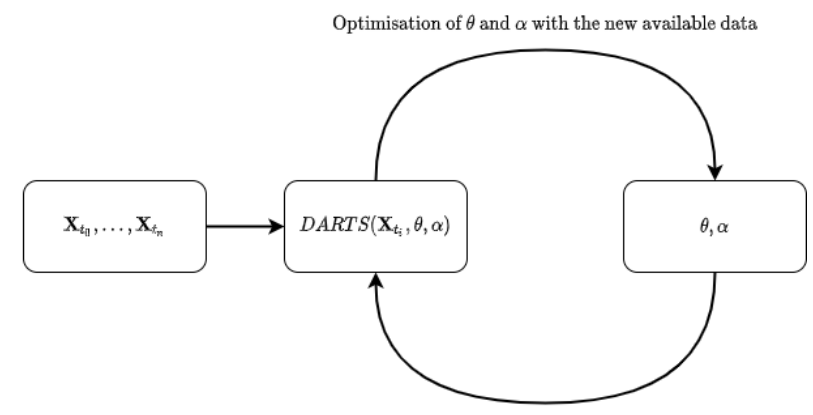

Use the new $\theta$ and $\alpha$ as the warmed-up initial state

Figure 2: Training procedure of ONAS. As new data $\mathrm{X}_{t_{i}}$ is coming, the neural network parameters, $\theta$ and $\alpha$ are updated using a DARTS optimisation scheme starting at the previously optimised supernet.

If we apply this process directly, there is a risk that the change between two consecutive architectures is too big and create a catastrophic forgetting event. To avoid this, we propose to use a learning rate correlated with the domain discrepancy between two consecutive batches from $T$. Domain discrepancy metrics measure how much an initial distribution varies from a target distribution. Different metrics exist and one goal of this research should be to determine a good metric to use in this online neural architecture search setting. A good initial metric to try would be the joint maximum mean discrepancy (joint MMD) [20] as it is a classical domain adaptation criterion.

\section{OUTLOOK}

The method we present in this work should be understood as a framework to deal with evolving data distributions with deep learning and as such, it would be of utmost interest to try different NAS methods and to adapt them to an online context. We chose DARTS as it is one of the quickest NAS algorithms and it has been shown to provide good results but there is a lot of potential in using other methods as well. As such, this proposal is not only about a precise algorithm that could work to solve a specific problem, but it is more about the creation of a framework to solve this problem. The specific algorithms used in that framework are yet to be determined given different use cases and data availability.

The main intention of this proposal is to make the community aware of the potential problem of evolving distributions, with change in the distribution complexity, and our proposal solution should just be seen as one of potentially many solutions to it. Furthermore, the first goal of this research should be to analyse and find more precisely under which conditions in the change of distribution would ONAS be a better alternative than the use of traditional NAS method with regular re-training of the best found architecture. The second goal would be to implement and test the proposed approach on a set of interesting datasets to find the real gain this approach can have. This will pose the mathematical and experimental basis upon which further work could be built and start the field of online architecture search to provide high impact research.

\section{REFERENCES}

[1] Agarwal, S., Vijaya Saradhi, V., And Karnick, H. Kernel-based online machine learning and support vector reduction. Neurocomputing 71, 7 (Mar. 2008), 12301237.

[2] Bello, I., Zoph, B., Vasudevan, V., ANd Le, Q. V. Neural Optimizer Search with Reinforcement Learning. arXiv:1709.07417 [cs, stat] (Sept. 2017). arXiv: 1709.07417.

[3] CaI, H., Zhu, L., ANd Han, S. ProxylessNAS: Direct Neural Architecture Search on Target Task and Hardware. arXiv:1812.00332 [cs, stat] (Feb. 2019). arXiv: 1812.00332.

[4] Casale, F. P., Gordon, J., And Fusi, N. Probabilistic Neural Architecture Search. arXiv:1902.05116 [cs, stat] (Feb. 2019). arXiv: 1902.05116.

[5] Chen, X., Xie, L., Wu, J., And Tian, Q. Progressive Differentiable Architecture Search: Bridging the Depth Gap Between Search and Evaluation. pp. 1294-1303.

[6] Chen, Y., Meng, G., Zhang, Q., Xiang, S., Huang, C., Mu, L., and Wang, X. RENAS: Reinforced Evolutionary Neural Architecture Search. pp. 4787-4796.

[7] Dong, X., AND YANG, Y. NAS-Bench-201: Extending the Scope of Reproducible Neural Architecture Search. arXiv:2001.00326 [cs] (Jan. 2020). arXiv: 2001.00326.

[8] Elsken, T., Metzen, J. H., And Hutter, F. Efficient Multi-objective Neural Architecture Search via Lamarckian Evolution. arXiv:1804.09081 [cs, stat] (Feb. 2019). arXiv: 1804.09081.

[9] Floreano, D., Dürr, P., ANd Mattiussi, C. Neuroevolution: from architectures to learning. Evolutionary Intelligence 1, 1 (Mar. 2008), 47-62.

[10] Fontenla-Romero, , Guijarro-Berdiñas, B., Martinez-Rego, D., PérezSÁnchez, B., And Peteiro-Barral, D. Online machine learning. In Efficiency and Scalability Methods for Computational Intellect. IGI Global, 2013, pp. 27-54.

[11] Gao, Q., Luo, Z., AND KLABJAn, D. Efficient Architecture Search for Continual Learning. arXiv:2006.04027 [cs, stat] (June 2020). arXiv: 2006.04027.

[12] Guo, Z., Zhang, X., Mu, H., Heng, W., Liv, Z., Wei, Y., And Sun, J. Single Path One-Shot Neural Architecture Search with Uniform Sampling. In Computer Vision - ECCV 2020, A. Vedaldi, H. Bischof, T. Brox, and J.-M. Frahm, Eds., vol. 12361. Springer International Publishing, Cham, 2020, pp. 544-560. Series Title: Lecture Notes in Computer Science.

[13] Huang, S., François-Lavet, V., and Rabusseau, G. Neural Architecture Search for Class-incremental Learning. arXiv:1909.06686 [cs, stat] (Sept. 2019). arXiv: 1909.06686.

[14] JiA, L., ZhaO, Q., AND Tong, L. Retail pricing for stochastic demand with unknown parameters: An online machine learning approach. In 2013 51st Annual Allerton Conference on Communication, Control, and Computing (Allerton) (Oct. 2013), pp. 1353-1358.

[15] Li, Y., Zhou, Y., WANG, Y., ANd TANG, Z. PD-DARTS: Progressive Discretization Differentiable Architecture Search. In Pattern Recognition and Artificial Intelligence, Y. Lu, N. Vincent, P. C. Yuen, W.-S. Zheng, F. Cheriet, and C. Y. Suen, Eds., vol. 12068. Springer International Publishing, Cham, 2020, pp. 306-311. Series Title: Lecture Notes in Computer Science.

[16] Liang, H., Zhang, S., Sun, J., He, X., Huang, W., Zhuang, K., and Li, Z. DARTS+: Improved Differentiable Architecture Search with Early Stopping. arXiv:1909.06035 [cs] (Oct. 2020). arXiv: 1909.06035.

[17] Liv, H., Long, M., WANG, J., AND WANG, Y. Learning to Adapt to Evolving Domains. Advances in Neural Information Processing Systems 33 (2020).

[18] Liu, H., Simonyan, K., ANd YANG, Y. DARTS: Differentiable Architecture Search. arXiv:1806.09055 [cs, stat] (Apr. 2019). arXiv: 1806.09055.

[19] Liu, J., Zhou, S., Wu, Y., Chen, K., Ouyang, W., And Xu, D. Block Proposal Neural Architecture Search. IEEE transactions on image processing: a publication of the IEEE Signal Processing Society PP (Oct. 2020).

[20] Long, M., Zhu, H., WANG, J., AND Jordan, M. I. Deep Transfer Learning with Joint Adaptation Networks. In International Conference on Machine Learning (July 2017), PMLR, pp. 2208-2217. ISSN: 2640-3498.

[21] Lu, Z., Deb, K., Goodman, E., Banzhaf, W., And Boddeti, V. N. NSGANetV2: Evolutionary Multi-Objective Surrogate-Assisted Neural Architecture Search. arXiv:2007.10396 [cs] (July 2020). arXiv: 2007.10396.

[22] Lukasik, J., Friede, D., Zela, A., Stuckenschmidt, H., Hutter, F., and Keuper, M. Smooth Variational Graph Embeddings for Efficient Neural Architecture Search. arXiv:2010.04683 [cs, stat] (Oct. 2020). arXiv: 2010.04683.

[23] Macko, V., Weill, C., Mazzawi, H., and Gonzalvo, J. Improving Neural Architecture Search Image Classifiers via Ensemble Learning. arXiv:1903.06236 [cs, stat] (Mar. 2019). arXiv: 1903.06236.

[24] Park, K.-M., Shin, D., AND CHI, S.-D. Variable Chromosome Genetic Algorithm for Structure Learning in Neural Networks to Imitate Human Brain. Applied Sciences 9, 15 (Aug. 2019), 3176.

[25] Pham, H., Guan, M. Y., Zoph, B., Le, Q. V., and Dean, J. Efficient Neural Architecture Search via Parameter Sharing. arXiv:1802.03268 [cs, stat] (Feb. 2018). arXiv: 1802.03268 . 
[26] Real, E., Aggarwal, A., Huang, Y., and Le, O. V. Regularized Evolution for Image Classifier Architecture Search. Proceedings of the AAAI Conference on Artificial Intelligence 33, 01 (July 2019), 4780-4789. Number: 01.

[27] Siems, J., Zimmer, L., Zela, A., Lukasik, J., Keuper, M., and Hutter, F. NASBench-301 and the Case for Surrogate Benchmarks for Neural Architecture Search. arXiv:2008.09777 [cs] (Nov. 2020). arXiv: 2008.09777.

[28] Stanley, K. O., Clune, J., Lehman, J., and Mirkkulainen, R. Designing neural networks through neuroevolution. Nature Machine Intelligence 1, 1 (Jan. 2019), 24-35. Number: 1 Publisher: Nature Publishing Group.

[29] Stanley, K. O., AND Mirkulainen, R. Evolving neural networks through augmenting topologies. Evolutionary Computation 10, 2 (June 2002), 99-127.

[30] Weng, Y., Zhou, T., Liu, L., And XiA, C. Automatic Convolutional Neural Architecture Search for Image Classification Under Different Scenes. IEEE Access 7 (2019), 38495-38506. Conference Name: IEEE Access.

[31] White, C., Neiswanger, W., and Savani, Y. BANANAS: Bayesian Optimization with Neural Architectures for Neural Architecture Search. arXiv:1910.11858 [cs, stat] (Nov. 2020). arXiv: 1910.11858

[32] Wu, B., Dai, X., Zhang, P., Wang, Y., Sun, F., Wu, Y., Tian, Y., Vajda, P., JiA Y., AND KeutZer, K. FBNet: Hardware-Aware Efficient ConvNet Design via Differentiable Neural Architecture Search. pp. 10734-10742.

[33] XiE, S., Zheng, H., Liu, C., AND Lin, L. SNAS: Stochastic Neural Architecture Search. arXiv:1812.09926 [cs, stat] (Mar. 2020). arXiv: 1812.09926.
[34] Xu, Y., Xie, L., Zhang, X., Chen, X., Oi, G.-J., Tian, O., and Xiong, H. PCDARTS: Partial Channel Connections for Memory-Efficient Architecture Search. arXiv:1907.05737 [cs] (Apr. 2020). arXiv: 1907.05737.

[35] YANG, Y., Li, H., You, S., WANG, F., Qian, C., AND Lin, Z ISTA-NAS: Efficient and Consistent Neural Architecture Search by Sparse Coding. arXiv:2010.06176 [cs] (Oct. 2020). arXiv: 2010.06176

[36] Yang, Z., Wang, Y., Chen, X., Shi, B., Xu, C., Xu, C., Tian, Q., And Xu, C. CARS: Continuous Evolution for Efficient Neural Architecture Search. arXiv:1909.04977 [cs] (Mar. 2020). arXiv: 1909.04977.

[37] Ying, C., Klein, A., Christiansen, E., Real, E., Murphy, K., And Hutter, F. NASBench-101: Towards Reproducible Neural Architecture Search. In International Conference on Machine Learning (May 2019), PMLR, pp. 7105-7114. ISSN: 26403498.

[38] Zela, A., Elsken, T., Saikia, T., Marrakchi, Y., Brox, T., and Hutter, F. Understanding and Robustifying Differentiable Architecture Search. arXiv:1909.09656 [cs, stat] (Jan. 2020). arXiv: 1909.09656.

[39] Zela, A., Siems, J., ANd Hutter, F. NAS-Bench-1Shot1: Benchmarking and Dissecting One-shot Neural Architecture Search. arXiv:2001.10422 [cs, stat] (Apr. 2020). arXiv: 2001.10422.

[40] Zhou, D., Zhou, X., Zhang, W., Loy, C. C., Yi, S., Zhang, X., And Ouyang, W. EcoNAS: Finding Proxies for Economical Neural Architecture Search. pp. 1139611404. 